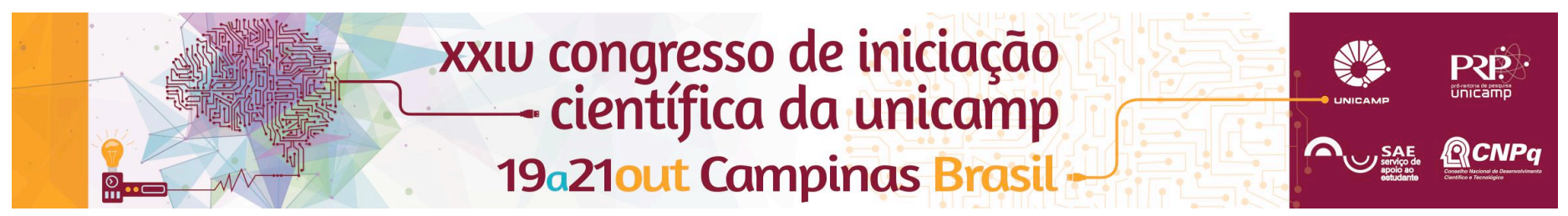

\title{
Analysis of copy number variation in the SCN1A gene in patients with Epileptic Encephalopathies and Generalized Epilepsy with Febrile Seizures Plus
}

\section{Caroline Magalhães de Toledo*, Camila V. Soler, Marina C. Gonsales, Iscia Lopes-Cendes}

\begin{abstract}
This research aims to assess copy number variations in patients with Childhood Epileptic Encephalopathies and Generalized Epilepsy with Febrile Seizures Plus using Multiplex Ligation Probe Amplification.
\end{abstract}

\author{
Key words: \\ Encephalopathy, SCN1A, Copy Number Variation
}

\section{Introduction}

Epileptic encephalopathy is a chronic disorder that consists of repeated disruptions in the electrical activity of the brain, which can culminate on psychomotor dysfunctions [1].

Associated issues of the Childhood Epileptic

Encephalopathies (CEE) are neurological, intellectual, behavioral or psychiatric disorders [2]. Since CEEs are complex disorders, it is a challenge to confirm these conditions with molecular findings. As some patients with Dravet Syndrome were found to have copy number variations in the SCN1A gene [3], one of the most important genes in the molecular analysis of epileptic encephalopathies, testing patients with other encephalopathies and clinically related conditions such as Generalized (or Genetic) Epilepsy with Febrile Seizures Plus (GEFS+) is essential. GEFS+ is an inherited condition that consists of repeated febrile seizures, usually generalized, that either continue after the age of 6 or also present afebrile seizures [4]. Most people with GEFS+ do not have cognitive deficit; however, a few patients with more severe presentation may have intellectual and physical disorders [5].

\section{Results and Discussion}

The technique used to assess copy number variations was Multiplex Ligation Probe Amplification (MLPA). The results of the MLPA were then evaluated in the computer program Coffalyzer. Samples of DNA from a total of 55 patients were analyzed though this technique, 48 of which were diagnosed with CEEs and 7 were diagnosed with GEFS+.

One patient with GEFS+ and borderline Dravet syndrome was found to have a deletion in exons 21 to 26 of the $S C N 1 A$ gene. It is predicted that this mutation is deleterious to protein function, since significant domains would be disrupted. This is an ongoing study, therefore, additional patients are being analyzed.
Image 1. Illustration of probe ratios in the single patient with deletion in SCN1A. For reference, it was adopted as normal the interval from ratios $0.7-1.3$, marked by the two red horizontal lines. Dots outside the normal interval depict the region where the deletion was found.

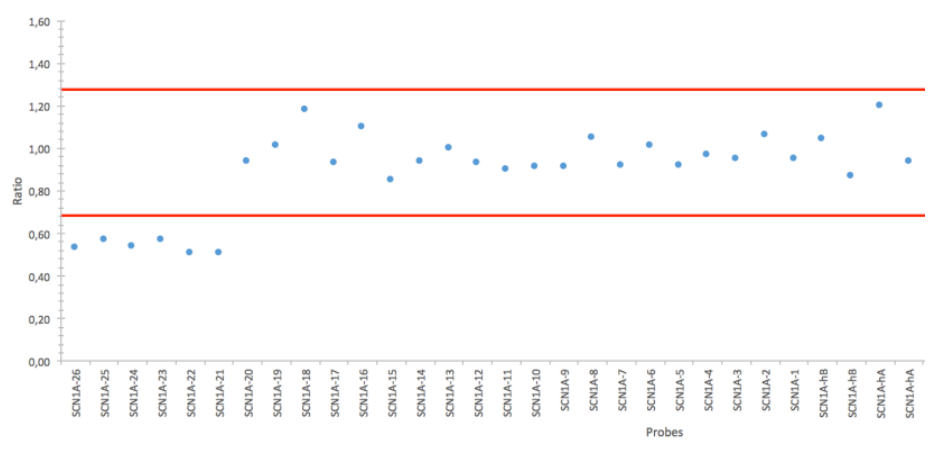

The deletion found in this work is possibly associated with the phenotype of the patient, who was diagnosed with borderline Dravet Syndrome. The analysis of the patient family history shows additional family members with febrile seizures, indicating the most likely diagnosis of GEFS+.

\section{Conclusions}

This work reinforced the importance of analyzing copy number variations, not only point mutations, in patients with CEEs other than Dravet Syndrome, including patients with GEFS+. Therefore, we can conclude that the clinical spectrum of microrearrangements in $S C N 1 A$ is broader than previously described.

[1] PANAYIOTOPOUlOS, C.P., "The Epilepsies - Seizures, Syndromes and Management", 2005; [2] CAMFIELD, P. R., CAMFIELD, C. S., Pediatric Neurology 51:17-23, 2014; [3] GONSALES, M. C. et al., Arq Neuropsiquiatr 73 (11):1-13, 2015;[4] SCHEFFER, I. E., BERKOVIC, S. F., Brain 120: 479490, 1997; [5] CAMFIELD, P. R., CAMFIELD, C. S., Epileptic Disord 17 (2): $124-133,2015$. 